\title{
Hinokitiol inhibits vasculogenic mimicry activity of breast cancer stem/progenitor cells through proteasome-mediated degradation of epidermal growth factor receptor
}

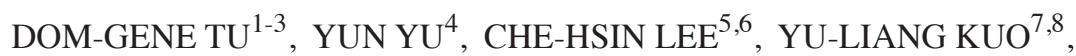 \\ YIN-CHE LU ${ }^{9}$, CHI-WEN TU ${ }^{10}$ and WEN-WEI CHANG ${ }^{4,11}$
}

\begin{abstract}
${ }^{1}$ Department of Nuclear Medicine, Ditmanson Medical Foundation Chia-Yi Christian Hospital, Chiayi 60002;
${ }^{2}$ Department of Food Science and Technology, Chia Nan University of Pharmacy \& Science, Tainan 717;

${ }^{3}$ Graduate Institute of Medical Sciences, College of Health Sciences, Chang Jung Christian University, Tainan 71101;

${ }^{4}$ School of Biomedical Sciences, College of Medical Science and Technology, Chung Shan Medical University,

Taichung 40201; ${ }^{5}$ Graduate Institute of Basic Medical Science; ${ }^{6}$ Department of Microbiology, School of Medicine, China Medical University, Taichung 40402; ${ }^{7}$ Department of Medical Imaging, Chung Shan Medical University Hospital;

${ }^{8}$ School of Medical Imaging and Radiological Sciences, Chung Shan Medical University, Taichung 40201;

${ }^{9}$ Division of Hematology-Oncology; ${ }^{10}$ Department of Surgery, Ditmanson Medical Foundation Chia-Yi Christian Hospital, Chiayi 60002; ${ }^{11}$ Department of Medical Research, Chung Shan Medical University Hospital, Taichung 40201, Taiwan, R.O.C.
\end{abstract}

Received January 12, 2015; Accepted February 11, 2016

DOI: $10.3892 / \mathrm{ol} .2016 .4300$

\begin{abstract}
Hinokitiol, alternatively known as $\beta$-thujaplicin, is a tropolone-associated natural compound with antimicrobial, anti-inflammatory and antitumor activity. Breast cancer stem/progenitor cells (BCSCs) are a subpopulation of breast cancer cells associated with tumor initiation, chemoresistance and metastatic behavior, and may be enriched by mammosphere cultivation. Previous studies have demonstrated that BCSCs exhibit vasculogenic mimicry (VM) activity via the epidermal growth factor receptor (EGFR) signaling pathway. The present study investigated the anti-VM activity of hinokitiol in BCSCs. At a concentration below the half maximal inhibitory concentration, hinokitiol inhibited VM formation of mammosphere cells derived from two human breast cancer cell lines. Hinokitiol was additionally indicated to downregulate EGFR protein expression in mammosphere-forming BCSCs without affecting the expression of messenger RNA. The protein stability of EGFR in BCSCs was also decreased by hinokitiol. The EGFR protein expression and VM formation capability of
\end{abstract}

Correspondence to: Professor Wen-Wei Chang, School of Biomedical Sciences, College of Medical Science and Technology, Chung Shan Medical University, 110 Chien-Kuo North Road, Taichung 40201, Taiwan, R.O.C.

E-mail: changww@csmu.edu.tw

Abbreviations: VM, vasculogenic mimicry; BCSCs, breast cancer stem/progenitor cells; EGFR, epidermal growth factor receptor

Key words: hinokitiol, vasculogenic mimicry, breast cancer stem/progenitor cells, epidermal growth factor receptor hinokitiol-treated BCSCs were restored by co-treatment with MG132, a proteasome inhibitor. In conclusion, the present study indicated that hinokitiol may inhibit the VM activity of BCSCs through stimulating proteasome-mediated EGFR degradation. Hinokitiol may act as an anti-VM agent, and may be useful for the development of novel breast cancer therapeutic agents.

\section{Introduction}

Cancer stem/progenitor cells (CSCs) are a subpopulation of cancer cells with the characteristics of tumor initiation (1), resistance to therapy (2) and metastasis (3). In breast cancer, breast CSCs (BCSCs) have been identified as cells with cluster of differentiation (CD) $24{ }^{-} \mathrm{CD} 44^{+}$surface markers (4) or with high intracellular aldehyde dehydrogenase activity (5). BCSCs may additionally be enriched by cultivation in a serum-free, non-adherent environment, known as the mammosphere, which is a floating clump composed of breast cancer cells $(6,7)$. In addition to tumor initiation and chemoresistant properties, BCSCs have also been indicated to exhibit vasculogenic mimicry (VM) activity (8), defined as the formation of perfusable, matrix-rich and vasculogenic-like networks by tumor cells, without involvement of endothelial cells (9). A previous study demonstrated that the VM activity of BCSCs is regulated by epidermal growth factor receptor (EGFR) signaling (8).

Hinokitiol, alternatively known as $\beta$-thujaplicin, is a tropolone-associated natural compound isolated from heartwood cupressaceous plants (10), and has been widely used as an antimicrobial agent in toothpastes, cosmetics and food (11). In addition to antimicrobial activity, hinokitiol has been reported to possess anti-inflammatory $(12,13)$ and antitumor 
activity $(14,15)$. Previously, in prostate carcinoma cell lines, hinokitiol was indicated to disrupt androgen receptor signaling and inhibit cell growth (14). Hinokitiol may induce G1 arrest in malignant melanoma cells through increased cyclin-dependent kinase inhibitor 1B protein expression (15). Hinokitiol was also indicated to cause caspase-dependent apoptosis (16) and differentiation (17) in teratocarcinoma F9 cells. A recent study indicated that hinokitiol induced autophagy in murine breast and colorectal cancer cells via downregulation of the v-akt murine thymoma viral oncogene homolog $1 /$ mechanistic target of rapamycin (serine/threonine kinase) signaling pathway, which led to cell death (18). Although the anti-tumor activity of hinokitiol has been reported, its effect on CSCs remains to be elucidated.

The present study indicated that hinokitiol may inhibit the VM activity of BCSCs enriched by mammosphere cultivation at a concentration below the half maximal inhibitory concentration $\left(\mathrm{IC}_{50}\right)$. Hinokitiol was also indicated to decrease the protein expression of EGFR without affecting the expression of messenger (m)RNA. In addition, downregulation of EGFR protein by hinokitiol was mediated through proteasome degradation. Inhibited proteasome activity by MG132 abolished the anti-VM activity of hinokitiol. The results of the present study suggest that hinokitiol may act as an anti-VM agent, and may be useful for the development of novel breast cancer therapeutic agents

\section{Materials and methods}

Cell culture and reagents. The MDA-MB-231 human breast cancer cell line was obtained from American Type Culture Collection (ATCC; Manassas, VA, USA) and maintained according to ATCC's recommendations. The AS-B244 human breast cancer cell line was established from the tissue of a female patient (Academia Sinica, Taipei, Taiwan) with triple negative breast cancer and maintained as previously described (19). Hinokitiol was purchased from Sigma-Aldrich (St. Louis, MO, USA), dissolved in ethanol (EtOH; Avantor Performance Materials, Center Valley, PA, USA) as a $100 \mathrm{mM}$ stock solution and stored at $-20^{\circ} \mathrm{C}$. MG132 was purchased from Tocris Bioscience (Bristol, United Kingdom), dissolved in dimethyl sulfoxide (Sigma-Aldrich) as a $100 \mathrm{mM}$ stock solution and stored at $-20^{\circ} \mathrm{C}$.

Enrichment of BCSCs by mammosphere cultivation. Cells were resuspended in Dulbecco's Modified Eagle's Medium: Nutrient Mixture F-12 (Thermo Fisher Scientific Inc., Waltham, MA, USA) containing 1\% methyl cellulose (Sigma-Aldrich) to avoid cell aggregation, fibroblast growth factor-basic (20 ng/ml; PeproTech, Inc., Rocky Hill, NJ, USA), human epidermal growth factor (20 ng/ml; PeproTech, Inc.,), insulin $(5 \mu \mathrm{g} / \mathrm{ml}$; Sigma-Aldrich) and B27 supplement (dilution, 1:50; Gibco ${ }^{\circledR}$; Thermo Fisher Scientific, Inc.). Cells were seeded at $10^{4}$ cells per dish into ultralow-attachment $10 \mathrm{~cm}$ dishes (Corning Life Sciences BV, Amsterdam, Netherlands). Following 7 days of incubation, the mammospheres were collected using a $100 \mathrm{~mm}$ cell strainer (BD Biosciences, San Jose, CA, USA) and dissociated by HyQTase (Hyclone; GE Healthcare, Logan, UT, USA) to achieve a single cell suspension for additional experiments.
Cytotoxicityanalysis. For the cytotoxicity assay, $1 \times 10^{4}$ mammosphere cells/well were seeded in 96-well plates (Corning Life Sciences BV) and cultured for $48 \mathrm{~h}$ with or without hinokitiol or $0.1 \%$ EtOH. Cell viability was determined by WST-1 reagent (BioVision, Inc., Milpitas, CA, USA) using a microplate reader (SpectraMax Plus 384; Molecular Devices, LLC, Sunnyvale, CA, USA), according to the manufacturer's protocol. The $\mathrm{IC}_{50}$ value was calculated by GraFit version 7 software (Erithacus Software Ltd., East Grinstead, UK).

In vitro VM activity assay. Wells of $\mu$-microslide (ibidi $\mathrm{GmbH}$, Martinsried, Germany) were coated with $10 \mu$ l of Matrigel (BD Biosciences) at a concentration of $8 \mathrm{mg} / \mathrm{ml}$, and incubated at $37^{\circ} \mathrm{C}$ overnight. Mammosphere cells were suspended at $2 \times 10^{4}$ cells $/ 50 \mu \mathrm{l}$ in M200 medium (Gibco; Thermo Fisher Scientific, Inc.) containing $1 \mathrm{X}$ low serum growth supplement (Gibco; Thermo Fisher Scientific, Inc.) and loaded into one Matrigel-coated well. The slide was then incubated at $37^{\circ} \mathrm{C}$ in a $5 \% \mathrm{CO}_{2}$ incubator and the $\mathrm{VM}$ structures were recorded by inverted microscopy (AE30; Motic Electric Group Co., Ltd., Xiamen, China) at $6 \mathrm{~h}$ post-seeding. Images of the wells were analyzed with the Tubeness function on Image J version $1.50 \mathrm{e}$ software (National Institutes of Health, Bethesda, MA, USA), and VM scores were calculated according to a formula described by Aranda et al (20) as follows: VM = [(no.spouting cells x 1) + (no. connected cells x 2) + (no. polygons x 3)] / total no. cells.

Western blot analysis. Cells were harvested using trypsin (Gibco; Thermo Fisher Scientific, Inc.), lysed in mammalian protein extraction reagent (Pierce; Thermo Fisher Scientific Inc.) and the protein concentration was determined by bicinchoninic acid reagent (Pierce; Thermo Fisher Scientific Inc.). In total, $25 \mu \mathrm{g}$ of extracted protein was separated by $10 \%$ sodium dodecyl sulfate-polyacrylamide gel electrophoresis and transferred to a polyvinylidene difluoride membrane (Immobilon-P; Merck Millipore, Darmstadt, Germany). The membranes were then blocked with $5 \%$ skimmed milk (Sigma-Aldrich), dissolved in Tris-buffered saline with $0.05 \%$ Tween-20 (TBST; Sigma-Aldrich) at room temperature for $1 \mathrm{~h}$, followed by incubation with primary antibodies [mouse anti-human EGFR monoclonal antibody was purchased from Santa Cruz Biotechnology Inc. (Dallas, TX, USA; catalog no. sc-377229); mouse anti-human tubulin monoclonal antibody was purchased from Novus Biologicals, LLC (Littleton, CO, USA; catalog no. NB100-690)] at $4^{\circ} \mathrm{C}$ overnight. Hydrogen peroxidase-conjugated anti-rabbit or anti-mouse immunoglobulin G polyclonal antibody (catalog no 7076; Cell Signaling Technology, Inc., Danvers, MA, USA) were used as secondary antibodies. The membranes were washed 3 times for 5 min each time with TBST following blocking, primary antibody incubation and secondary antibody incubation. Developed chemiluminescence signals from catalyzed ECL substrate (PerkinElmer Inc., Waltham, MA, USA) were detected by a Luminescence-Image Analyzer (ImageQuant LAS 4000 mini; GE Healthcare Bio-sciences, Pittsburgh, PA, USA).

Reverse transcription-quantitative polymerase chain reaction $(R T-q P C R)$. Total RNA was extracted from AS-B244 and MDA-MB-231 cells and purified using an RNA extraction 

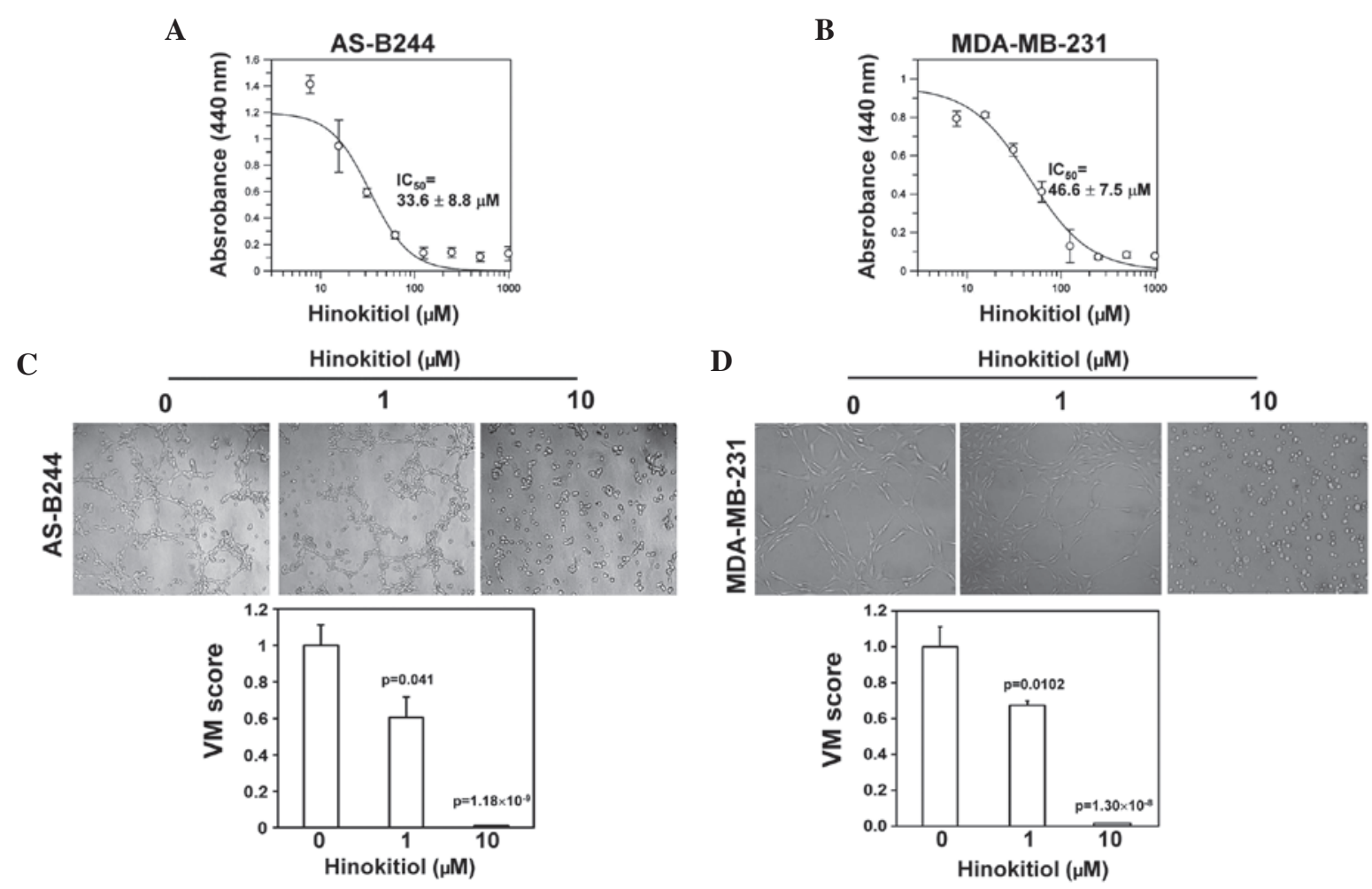

Figure 1. Hinokitiol inhibits VM activity of breast cancer stem/progenitor cells. Mammosphere cells derived from (A) AS-B244 or (B) MDA-MB-231 human breast cancer cells were treated with various concentrations of hinokitiol for $48 \mathrm{~h}$, and cell viability was determined by WST-1 reagent. IC $\mathrm{C}_{50}$ value was calculated with GraFit software. Mammosphere cells derived from (C) AS-B244 or (D) MDA-MB-231 cells were treated with 0.1\% ethanol or hinokitiol (1 or $10 \mu \mathrm{M})$ for $24 \mathrm{~h}$ and then seeded into Matrigel-coated microwells for the VM activity assay. Images were captured at $4 \mathrm{~h}$ and used for analyzing VM scores. $\mathrm{VM}$, vasculogenic mimicry; $\mathrm{IC}_{50}$, half maximal inhibitory concentration.

kit (Quick-RNA ${ }^{\mathrm{TM}}$ MiniPrep kit; Zymo Research Corporation, Irvine, CA, USA) and complementary DNA (cDNA) was synthesized with a first strand cDNA synthesis kit (RevertAid First Strand cDNA Synthesis kit; Fermentas; Thermo Fisher Scientific, Inc.). The expression of genes was detected with specific primers and the KAPA SYBR ${ }^{\mathrm{TM}}$ fast qPCR kit (Kapa Biosystems, Inc., Wilmington, MA, USA) with the ABI StepOne ${ }^{\mathrm{TM}}$ Real-Time PCR System (Applied Biosystems; Thermo Fisher Scientific, Inc.). The primer sequences used in the present study were synthesized by Integrated DNA Technologies Pte., Ltd. (Singapore, China) and were as follows: EGFR, forward 5'-CAGCGCTACCTTGTC ATTCA-3' and reverse 5'-TGCACTCAGAGAGCTCAGGA-3'; mitochondrial ribosomal protein L19, forward 5'-GGGATT TGCATTCAGAGATCAG-3' and reverse 5'-GGAAGGGCA TCTCGTAAG-3'. The cycling conditions were as follows: $50^{\circ} \mathrm{C}$ for $2 \mathrm{~min}, 95^{\circ} \mathrm{C}$ for $10 \mathrm{~min}$, followed by 40 cycles of $95^{\circ} \mathrm{C}$ for $10 \mathrm{sec}$ and $60^{\circ} \mathrm{C}$ for $1 \mathrm{~min}$. The end-point used in quantification was calculated by the StepOne ${ }^{\mathrm{TM}}$ software (v2.2.2; Applied Biosystems; Thermo Fisher Scientific, Inc.), and the quantification cycle number (Cq value) for each analyzed sample was calculated. EGFR expression was normalized to MRPL19, which has been reported as one of the most stable internal control genes (21-23), to derive the change in Cq value $(\triangle \mathrm{Cq})$. The primer sequence for MRPL19 was as follows: Forward, 5'-GGGATTTGCATTCAGAGATCAG-3'; and reverse, 5'-GGAAGGGCATCTCGTAAG-3'. The relative gene expression differences between groups was calculated using 2- ${ }^{\Delta \Delta C q}$ (24). The PCR experiments were repeated three times.
Protein stability assay. Cells were seeded into a 12-well plate (Corning Life Sciences BV) at a density of $1 \times 10^{5}$ cells/well and incubated with $50 \mu \mathrm{g} / \mathrm{ml}$ cycloheximide (Sigma-Aldrich) for $1,3,6,9$ or $12 \mathrm{~h}$ with $0.1 \% \mathrm{EtOH}$ or $10 \mu \mathrm{M}$ hinokitiol. Cells were harvested with trypsin at 1,3,6,9 or $12 \mathrm{~h}$ post-seeding and the protein expression was detected by western blot analysis.

Statistical analysis. Quantitative data are presented as the mean \pm standard deviation. Comparisons between two groups were analyzed with the Student's $t$-test. Comparisons among multiple groups $(>3)$ were analyzed with one-way analysis of variance, and post-hoc tests were performed with Tukey Multiple Comparison analysis. $\mathrm{P}<0.05$ was considered to indicate a statistically significant difference.

\section{Results}

Hinokitiol dose-dependently inhibits VM activity of mammosphere cells derived from human breast cancer cell lines. Hinokitiol, alternatively known as $\beta$-thujaplicin, is a tropolone-associated natural compound with antimicrobial, anti-inflammatory and antitumor activity. The present study initially examined the cytotoxic effect of hinokitiol on mammosphere cells derived from AS-B244 or MDA-MB-231 human breast cancer cells. The results revealed that the $\mathrm{IC}_{50}$ values of hinokitiol for AS-B244 or MDA-MB-231 cells were $33.6 \pm 8.8$ and $46.6 \pm 7.5 \mu \mathrm{M}$, respectively (Fig. $1 \mathrm{~A}$ and B). Furthermore, the present study determined whether hinokitiol 
A

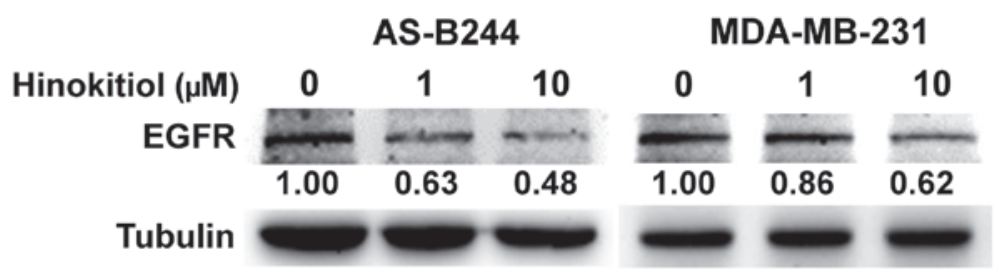

B
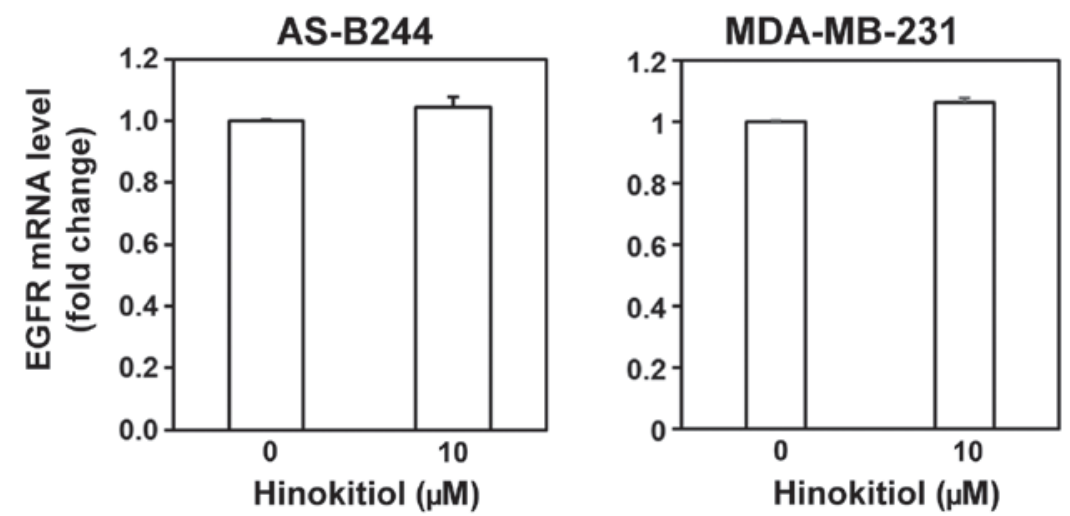

Figure 2. Hinokitiol suppresses the expression of EGFR protein in breast cancer stem/progenitor cells without affecting the mRNA. AS-B244 or MDA-MB231 mammosphere cells were treated with various concentrations of hinokitiol $(0$ and $10 \mu \mathrm{M})$ for $24 \mathrm{~h}$. (A) The expression of EGFR or tubulin protein was detected by western blot analysis. The number on the image is the relative expression level compared with $0.1 \%$ ethanol control. (B) The expression of EGFR mRNA was determined by reverse transcription-quantitative polymerase chain reaction. EGFR, epidermal growth factor receptor; mRNA, messenger RNA.

A

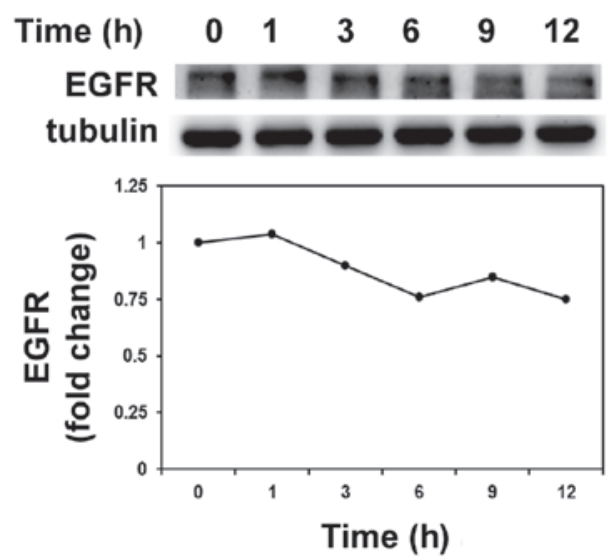

B
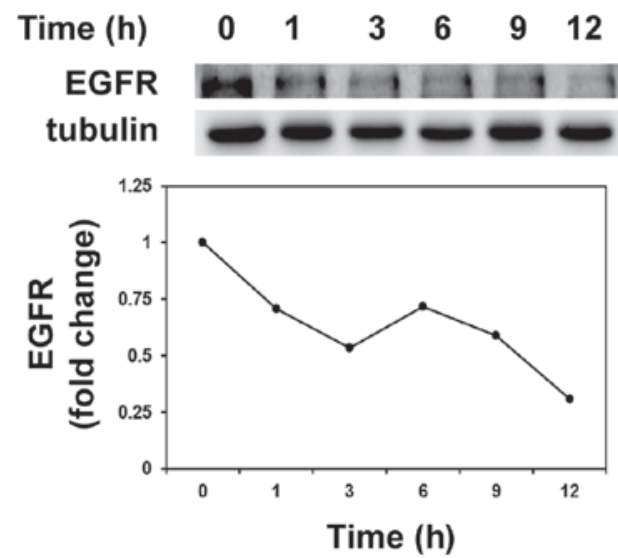

Figure 3. Hinokitiol decreases EGFR protein stability in AS-B244 mammosphere cells. AS-B244 mammosphere cells were treated with (A) 0.1\% ethanol or (B) $10 \mu \mathrm{M}$ hinokitiol in the presence of $100 \mu \mathrm{g} / \mathrm{ml}$ cyclohexamide, and total cell lysates were collected at various time points. The expression of EGFR protein was determined by western blot analysis and the data were presented as the fold change from the starting point ( 0 h). EGFR, epidermal growth factor receptor.

exhibited an inhibitory effect on the VM activity of BCSCs at concentrations below the $\mathrm{IC}_{50}$. The mammosphere cells derived from AS-B244 or MDA-MB-231 cells were treated with 1 or $10 \mu \mathrm{M}$ hinokitiol for $24 \mathrm{~h}$ and seeded into Matrigel-coated microwells for the analysis of in vitro VM activity in the presence of hinokitiol. Treatment with hinokitiol dose-dependently inhibited the VM activity of AS-B244 (Fig. 1C; $1 \mu \mathrm{M}, \mathrm{P}=0.041$; $10 \mu \mathrm{M}, \mathrm{P}=1.18 \times 10^{-9}$ ) or MDA-MB-231 (Fig. 1D; $1 \mu \mathrm{M}, \mathrm{P}=0.0102$; $\left.10 \mu \mathrm{M}, \mathrm{P}=1.30 \times 10^{-8}\right)$ mammosphere cells. These results indicate that hinokitiol may inhibit VM activity of BCSCs.

Hinokitiol inhibits EGFR expression without changing $m R N A$ expression. A previous study demonstrated that the VM activity of BCSCs is mediated by the activation of EGF/EGFR (8). The present study examined the effect of hinokitiol on EGFR expression. In the western blot analysis, the protein level of EGFR in AS-B244 or MDA-MB-231 mammosphere cells was downregulated in a dose-dependent manner by hinokitiol (Fig. 2A). The present study examined whether hinokitiol affected the mRNA expression of EGFR. In the RT-qPCR analysis, the mRNA level of EGFR in AS-B244 or MDA-MB-231 mammosphere cells did not change with hinokitiol treatment (Fig. 2B; AS-B244, P=0.1088; MDA-MB-231, $\mathrm{P}=0.0502$ ). Previous studies have reported that surface EGFR expression may be regulated by the proteasomal protein degradation pathway $(25,26)$. The present study examined the 
A

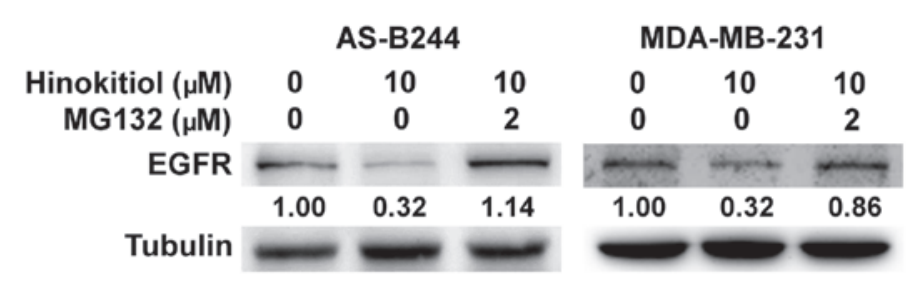

B

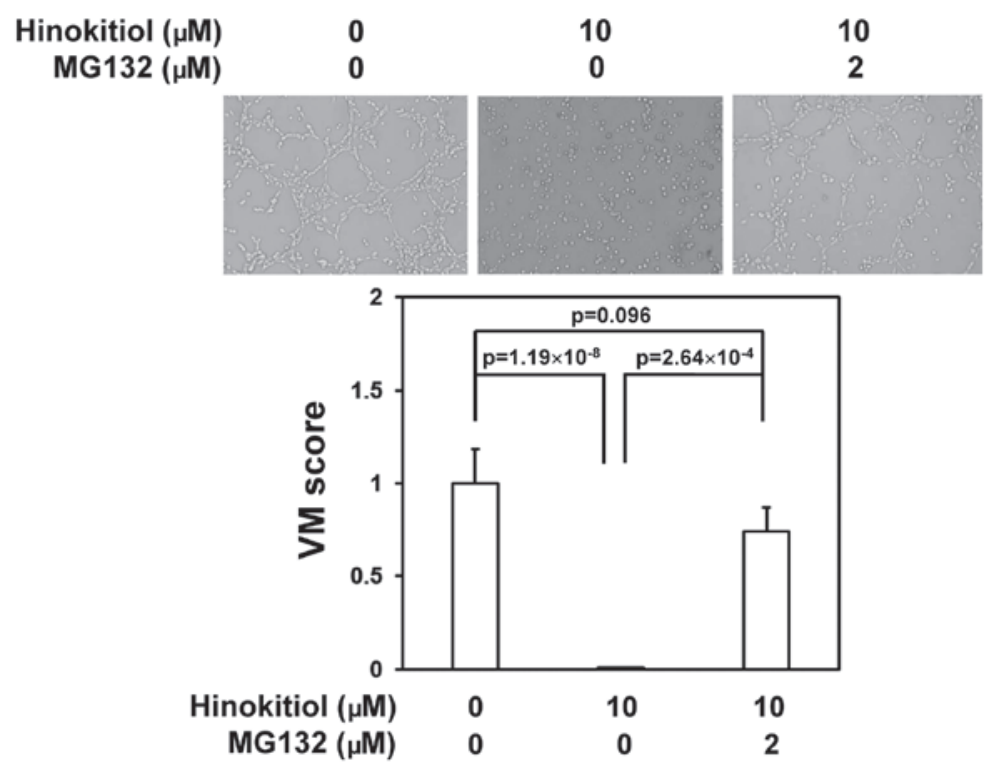

Figure 4. Hinokitiol inhibits EGFR expression in breast cancer stem/progenitor cells through proteasomal degradation. (A) AS-B244 or MDA-MB231 mammosphere cells were treated with $10 \mu \mathrm{M}$ hinokitiol or $10 \mu \mathrm{M}$ hinokitiol in the presence of $2 \mu \mathrm{M}$ MG132 for $24 \mathrm{~h}$. The expression of EGFR or tubulin protein was detected by western blot analysis. The number on the image is the relative expression level compared with the $0.1 \%$ ethanol control. (B) AS-B244 mammosphere cells were treated with $10 \mu \mathrm{M}$ hinokitiol or $10 \mu \mathrm{M}$ hinokitiol in the presence of $2 \mu \mathrm{M}$ MG132 for $24 \mathrm{~h}$ and cells were then harvested to determine the VM activity by seeding into Matrigel-coated microwells. Images were captured at $4 \mathrm{~h}$ post-seeding and VM scores were calculated with Image J software. EGFR, epidermal growth factor receptor; VM, vasculogenic mimicry.

protein stability of EGFR following hinokitiol treatment, and the results indicated that hinokitiol decreased EGFR protein stability in AS-B244 mammosphere cells (Fig. 3).

Hinokitiol inhibits EGFR expression via proteasome-mediated degradation. The present study determined whether the downregulation of EGFR protein expression by hinokitiol in BCSCs was mediated by the proteasomal degradation pathway. Following co-treatment in combination with MG132, a proteasome inhibitor, hinokitiol lost the ability to inhibit EGFR protein expression in AS-B244 or MDA-MB-231 mammosphere cells (Fig. 4A). The VM inhibition activity of hinokitiol in AS-B244 mammosphere cells was additionally reversed by MG132 treatment (Fig. 4B; $\mathrm{P}=2.64 \times 10^{-4}$ ). These results suggest that the $\mathrm{VM}$ inhibition activity of hinokitiol in breast cancer stem/progenitor cells may be mediated by proteasomal degradation of EGFR.

\section{Discussion}

Malignant tissues remain small in size (a few millimeters) when there is a lack of novel vasculature (27). One of the mechanisms of metastasis is the shedding of tumor cells into newly synthesized vessels (28). In breast cancer, the overexpression of vascular endothelial growth factor (VEGF), which is a key molecule for regulating angiogenesis (29), is associated with disease progression (30). Targeting VEGF signaling by antibodies or tyrosine kinase receptor inhibitors has been developed as a therapeutic strategy for several types of cancer (31). The monoclonal anti-VEGF antibody, bevacizumab, was approved for the treatment of certain types of colon, lung, kidney and brain cancer, but was subsequently announced to be unsafe and ineffective in breast cancer patients by the Food and Drug Administration of the USA in 2011 (32). One of the potential mechanisms for the poor effectiveness of bevacizumab in breast cancer was hypothesized to be due to VM (33). The development of novel agents that target VM may be important in the future of breast cancer therapy.

CSCs have been reported to be important in tumor vasculatures (34). CD $44^{+}$type I epithelial ovarian cancer cells have been demonstrated to form vascular structures when cultured in Matrigel conditions in vitro and to transdifferentiate into $\mathrm{CD} 4^{+}$endothelial progenitor cells (35). The progeny of glioma CSCs cultured in endothelial conditions have previously exhibited features of functional endothelial cells (36). In another study, blocking VEGF signaling suppressed the maturation of tumor endothelial progenitors into endothelium, and the inhibition of Notch signaling abolished the transition of glioma CSCs into endothelial progenitors (37). These previous studies suggest that CSCs may support tumor vascularization via direct transdifferentiation into endothelial cells 
or progenitors. A recent study indicated that BCSCs display VM activity in vivo and in vitro (8). Other studies have also demonstrated that the VM activity in mammosphere cells is derived from melanoma cells (38) or CD133+ ${ }^{+}$glioblastoma stem-like cells (39). These studies indicated that CSC-derived VM structures may be an important blood supply system in cancer. The present study demonstrated that hinokitiol, a tropolone-associated natural compound, may suppress the VM activity of BCSCs via the proteasomal degradation of EGFR. Previous studies have suggested that enhancing the degradation of EGFR may provide a promising approach in cancer treatment (25). It has additionally been demonstrated that hinokitiol is able to cause death of breast cancer cells via induction of autophagy (18). Huang et al (40) demonstrated that hinokitiol may inhibit the expression of matrix metalloprotease- 1 through the reduction of nuclear factor- $\kappa \mathrm{B}$ $(\mathrm{NF}-\kappa \mathrm{B})$ activation, resulting in the suppression of metastasis in a mouse melanoma model. Zhang et al (41) demonstrated that the anti-VM activity of thalidomide in melanoma was associated with the NF- $\kappa \mathrm{B}$ signaling pathway. The anti-VM activity of hinokitiol may also be mediated through an $\mathrm{NF}-\kappa \mathrm{B}$ associated signaling pathway, which requires additional investigation. The study conducted by Itzhaki et al (42) indicated that the simultaneous anti-cell growth and anti-VM activity of nicotinamide resulted in efficient anti-neoplastic effects in aggressive melanomas. Therefore, similar agents with anti-cell growth and anti-VM activity may act as potential anticancer compounds in the future development of breast cancer therapy.

In conclusion, the present study demonstrates that hinokitiol exhibits an inhibitory effect in VM activity of BCSCs through proteasomal degradation of EGFR. The anti-CSC activity of hinokitiol and the underling molecular mechanisms will be worthy of investigation in the future.

\section{Acknowledgements}

The present study was supported by an inter-institutional cooperation project between Chung Shan Medical University and Ditmanson Medical Foundation Chia-Yi Christian Hospital (Taichung, Taiwan; grant no. CSMU-CYC-102-01) and the Ministry of Science and Technology in Taiwan (grant nos. NSC-101-2314-040-015-MY2 and MOST-103-2314-B-040-015-MY3).

\section{References}

1. White AC and Lowry WE: Refining the role for adult stem cells as cancer cells of origin. Trends Cell Biol 25: 11-20, 2015.

2. O'Connor ML, Xiang D, Shigdar S, Macdonald J, Li Y, Wang T, Pu C, Wang Z, Qiao L and Duan W: Cancer stem cells: A contentious hypothesis now moving forward. Cancer Lett 344 $180-187,2014$.

3. Geng SQ, Alexandrou AT and Li JJ: Breast cancer stem cells: Multiple capacities in tumor metastasis. Cancer Lett 349: 1-7, 2014.

4. Al-Hajj M, Wicha MS, Benito-Hernandez A, Morrison SJ and Clarke MF: Prospective identification of tumorigenic breast cancer cells. Proc Natl Acad Sci USA 100: 3983-3988, 2003.

5. Ginestier C, Hur MH, Charafe-Jauffret E, Monville F, Dutcher J, Brown M, Jacquemier J, Viens P, Kleer CG, Liu S, et al: ALDH1 is a marker of normal and malignant human mammary stem cells and a predictor of poor clinical outcome. Cell Stem Cell 1: $555-567,2007$
6. Saadin K and White IM: Breast cancer stem cell enrichment and isolation by mammosphere culture and its potential diagnostic applications. Expert Rev Mol Diagn 13: 49-60, 2013.

7. Ponti D, Costa A, Zaffaroni N, Pratesi G, Petrangolini G, Coradini D, Pilotti S, Pierotti MA and Daidone MG: Isolation and in vitro propagation of tumorigenic breast cancer cells with stem/progenitor cell properties. Cancer Res 65: 5506-5511, 2005.

8. Lee CH, Wu YT, Hsieh HC, Yu Y, Yu AL and Chang WW: Epidermal growth factor/heat shock protein 27 pathway regulates vasculogenic mimicry activity of breast cancer stem/progenitor cells. Biochimie 104: 117-126, 2014.

9. Seftor RE, Hess AR, Seftor EA, Kirschmann DA, Hardy KM, Margaryan NV and Hendrix MJ: Tumor cell vasculogenic mimicry: From controversy to therapeutic promise. Am J Pathol 181: 1115-1125, 2012.

10. Jayakumar T, Hsu WH, Yen TL, Luo JY, Kuo YC, Fong TH and Sheu JR: Hinokitiol, a natural tropolone derivative, offers neuroprotection from thromboembolic stroke in vivo. Evid Based Complement Alternat Med 2013: 840487, 2013

11. Saeki Y, Ito Y, Shibata M, Sato Y, Okuda K and Takazoe I: Antimicrobial action of natural substances on oral bacteria. Bull Tokyo Dent Coll 30: 129-135, 1989.

12. Shih YH, Lin DJ, Chang KW, Hsia SM, Ko SY, Lee SY, Hsue SS, Wang TH, Chen YL and Shieh TM: Evaluation physical characteristics and comparison antimicrobial and anti-inflammation potentials of dental root canal sealers containing hinokitiol in vitro. PLoS One 9: e94941, 2014.

13. Shih MF, Chen LY, Tsai PJ and Cherng JY: In vitro and in vivo therapeutics of $\beta$-thujaplicin on LPS-induced inflammation in macrophages and septic shock in mice. Int J Immunopathol Pharmacol 25: 39-48, 2012.

14. Liu S and Yamauchi H: Hinokitiol, a metal chelator derived from natural plants, suppresses cell growth and disrupts androgen receptor signaling in prostate carcinoma cell lines. Biochem Biophys Res Commun 351: 26-32, 2006.

15. Liu S and Yamauchi H: P27-Associated G1 arrest induced by hinokitiol in human malignant melanoma cells is mediated via down-regulation of $\mathrm{pRb}$, Skp2 ubiquitin ligase and impairment of Cdk2 function. Cancer Lett 286: 240-249, 2009.

16. Ido Y, Muto N, Inada A, Kohroki J, Mano M, Odani T, Itoh N, Yamamoto K and Tanaka K: Induction of apoptosis by hinokitiol, a potent iron chelator, in teratocarcinoma F9 cells is mediated through the activation of caspase-3. Cell Prolif 32: 63-73, 1999.

17. Muto N, Dota A, Tanaka T, Itoh N, Okabe M, Inada A, Nakanishi T and Tanaka K: Hinokitiol induces differentiation of teratocarcinoma F9 cells. Biol Pharm Bull 18: 1576-1579, 1995.

18. Wang WK, Lin ST, Chang WW, Liu LW, Li TY, Kuo CY, Hsieh JL and Lee CH: Hinokitiol induces autophagy in murine breast and colorectal cancer cells. Environ Toxicol, 2014.

19. Chang WW, Lin RJ, Yu J, Chang WY, Fu CH, Lai A, Yu JC and Yu AL: The expression and significance of insulin-like growth factor-1 receptor and its pathway on breast cancer stem/progenitors. Breast Cancer Res 15: R39, 2013.

20. Aranda E and Owen GI: A semi-quantitative assay to screen for angiogenic compounds and compounds with angiogenic potential using the EA. hy926 endothelial cell line. Biol Res 42: 377-389, 2009.

21. Ayakannu T, Taylor AH, Willets JM, et al: Validation of endogenous control reference genes for normalizing gene expression studies in endometrial carcinoma. Mol Hum Reprod 21: 723-735, 2015.

22. Mohelnikova-Duchonova B, Oliverius M, Honsova E and Soucek P: Evaluation of reference genes and normalization strategy for quantitative real-time PCR in human pancreatic carcinoma. Dis Markers 32: 203-210, 2012.

23. McNeill RE, Miller N and Kerin MJ: Evaluation and validation of candidate endogenous control genes for real-time quantitative PCR studies of breast cancer. BMC Mol Biol 8: 107, 2007.

24. Livak KJ and Schmittgen TD: Analysis of relative gene expression data using real-time quantitative PCR and the 2(-Delta Delta C(T)) Method. Methods 25: 402-408, 2001.

25. Lipkowitz S: The role of the ubiquitination-proteasome pathway in breast cancer: Ubiquitin mediated degradation of growth factor receptors in the pathogenesis and treatment of cancer. Breast Cancer Res 5: 8-15, 2003.

26. Authier F, Métioui M, Bell AW and Mort JS: Negative regulation of epidermal growth factor signaling by selective proteolytic mechanisms in the endosome mediated by cathepsin B. J Biol Chem 274: 33723-33731, 1999. 
27. Folkman J: Tumor angiogenesis: Therapeutic implications. N Engl J Med 285: 1182-1186, 1971.

28. Schneider BP and Miller KD: Angiogenesis of breast cancer. J Clin Oncol 23: 1782-1790, 2005.

29. Hicklin DJ and Ellis LM: Role of the vascular endothelial growth factor pathway in tumor growth and angiogenesis. J Clin Oncol 23: 1011-1027, 2005.

30. Foekens JA, Peters HA, Grebenchtchikov N, Look MP, Meijer-van Gelder ME, Geurts-Moespot A, van der Kwast TH, Sweep CG and Klijn JG: High tumor levels of vascular endothelial growth factor predict poor response to systemic therapy in advanced breast cancer. Cancer Res 61: 5407-5414, 2001.

31. Hoeben A, Landuyt B, Highley MS, Wildiers H, Van Oosterom AT and De Bruijn EA: Vascular endothelial growth factor and angiogenesis. Pharmacol Rev 56: 549-580, 2004.

32. Sasich LD and Sukkari SR: The US FDAs withdrawal of the breast cancer indication for Avastin (bevacizumab). Saudi Pharm J 20: 381-385, 2012

33. Kirschmann DA, Seftor EA, Hardy KM, Seftor RE and Hendrix MJ: Molecular pathways: Vasculogenic mimicry in tumor cells: Diagnostic and therapeutic implications. Clin Cancer Res 18: 2726-2732, 2012.

34. Silvan U, Diez-Torre A, Bonilla Z, Moreno P, Diaz-Nunez M and Arechaga J: Vasculogenesis and angiogenesis in nonseminomatous testicular germ cell tumors. Urol Oncol 33: 268 e217-228, 2015.

35. Alvero AB, Fu HH, Holmberg J, Visintin I, Mor L, Marquina CC, Oidtman J, Silasi DA and Mor G: Stem-like ovarian cancer cells can serve as tumor vascular progenitors. Stem Cells 27: 2405-2413, 2009.
36. Ricci-Vitiani L, Pallini R, Biffoni M, Todaro M, Invernici G, Cenci T, Maira G, Parati EA, Stassi G, Larocca LM and De Maria R: Tumour vascularization via endothelial differentiation of glioblastoma stem-like cells. Nature 468: 824-828, 2010.

37. Wang R, Chadalavada K, Wilshire J, Kowalik U, Hovinga KE, Geber A, Fligelman B, Leversha M, Brennan C and Tabar V: Glioblastoma stem-like cells give rise to tumour endothelium. Nature 468: 829-833, 2010.

38. Monzani E and La Porta CA: Targeting cancer stem cells to modulate alternative vascularization mechanisms. Stem Cell Rev 4: 51-56, 2008.

39. Chiao MT, Yang YC, Cheng WY, Shen CC and Ko JL: CD133+ glioblastoma stem-like cells induce vascular mimicry in vivo. Curr Neurovasc Res 8: 210-219, 2011.

40. Huang $\mathrm{CH}$, Lu SH, Chang CC, Thomas PA, Jayakumar T and Sheu JR: Hinokitiol, a tropolone derivative, inhibits mouse melanoma (B16-F10) cell migration and in vivo tumor formation. Eur J Pharmacol 746: 148-157, 2015.

41. Zhang S, Li M, Gu Y, Liu Z, Xu S, Cui Y and Sun B: Thalidomide influences growth and vasculogenic mimicry channel formation in melanoma. J Exp Clin Cancer Res 27: 60, 2008.

42. Itzhaki O, Greenberg E, Shalmon B, Kubi A, Treves AJ, Shapira-Frommer R, Avivi C, Ortenberg R, Ben-Ami E, Schachter J, et al: Nicotinamide inhibits vasculogenic mimicry, an alternative vascularization pathway observed in highly aggressive melanoma. PLoS One 8: e57160, 2013. 